\title{
Le royaume de Bourgogne autour de l'an Mil
}

\section{Laurent Ripart}

\section{(2) OpenEdition}

\section{Journals}

\section{Édition électronique}

URL : https://journals.openedition.org/cem/11188

DOI : $10.4000 /$ cem. 11188

ISSN : 1954-3093

\section{Éditeur}

Centre d'études médiévales Saint-Germain d'Auxerre

\section{Édition imprimée}

Date de publication : 15 août 2009

Pagination : 411-415

ISSN : 1623-5770

\section{Référence électronique}

Laurent Ripart, «Le royaume de Bourgogne autour de l'an Mil », Bulletin du centre d'études médiévales d'Auxerre | BUCEMA [En ligne], 13 | 2009, mis en ligne le 09 septembre 2009, consulté le 22 septembre 2022. URL : http://journals.openedition.org/cem/11188; DOI : https://doi.org/10.4000/cem.11188

Ce document a été généré automatiquement le 22 septembre 2022.

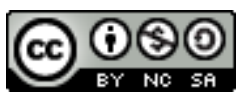

Creative Commons - Attribution - Pas d'Utilisation Commerciale - Partage dans les Mêmes Conditions 4.0 International - CC BY-NC-SA 4.0

https://creativecommons.org/licenses/by-nc-sa/4.0/ 


\section{Le royaume de Bourgogne autour de l'an Mil}

\section{Laurent Ripart}

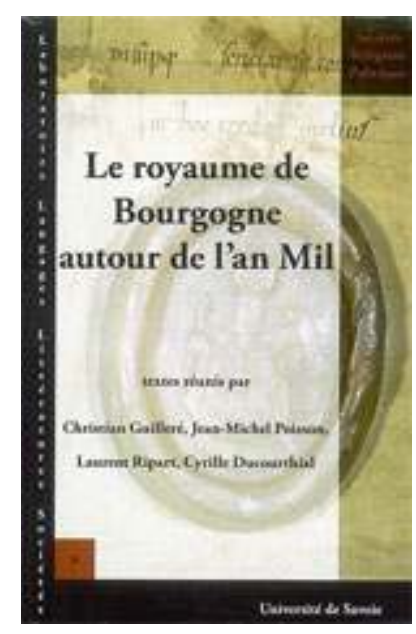

1 Aucun État moderne ou contemporain n'ayant jamais cherché à se situer dans l'héritage du royaume de Bourgogne, cette monarchie post-carolingienne n'a pas suscité un grand intérêt chez les historiens. Longtemps laissée aux bons soins des seuls érudits locaux, son histoire ne fut ainsi pour la première fois écrite qu'en 1901 et 1907, dans les deux ouvrages que René Poupardin consacra aux deux royaumes de Bourgogne $^{1}$ : celui, septentrional, des Rodolphiens, et celui, provençal, des Bosonides, qui fut annexé par le premier à une date que les historiens situent aujourd'hui autour de $942^{2}$. Aux côtés de Paul Fournier, René Parisot et Louis Jacob ${ }^{3}$, René Poupardin faisait partie de cette génération de jeunes et brillants historiens de l'école positiviste française, qui, aiguillée par son désir de revanche sur la défaite de 1870, s’interrogeait sur les frontières orientales de la France et le destin des terres "d'entre-deux ». Ils provoquèrent ainsi une polémique avec les historiens allemands, qui abordèrent de leur côté l'histoire du royaume de Bourgogne au seul prisme de ses relations avec l'Empire ${ }^{4}$. 
2 Ces recherches nationalistes ayant perdu de leur actualité avec la première guerre mondiale, le royaume de Bourgogne ne trouva dès lors plus guère d'historiens. À l'exception de quelques articles isolés ${ }^{5}$, ainsi que des thèses de Bernard Bligny et de Jean-Pierre Poly ${ }^{6}$, qui n'abordèrent toutefois l'histoire du royaume de Bourgogne que de manière très marginale, il n'y eut dans les décennies qui suivirent les publications de René Poupardin aucune nouvelle étude sur la monarchie bourguignonne. La remarquable édition des diplômes rodolphiens, que Theodor Schieffer donna en $1977^{7}$, suscita bien quelques travaux érudits ${ }^{8}$, mais elle ne put suffire à relancer les recherches sur le royaume de Bourgogne.

3 Ce n'est qu'à partir de la fin des années 1980 que les choses commencèrent à changer. Sous l'impulsion principale de Giuseppe Sergi, l'histoire du royaume de Bourgogne fit l'objet de plusieurs importantes contributions d'historiens issus de l'école de Turin ${ }^{9}$. Ce renouveau se poursuivit au cours des années 1990, par l'intermédiaire cette fois-ci de quelques travaux suisses ${ }^{10}$, mais aussi et surtout d'une série de thèses françaises de nouveau régime, toutes plus ou moins parues autour de 2000, qui étudiaient les différents espaces régionaux du royaume de Bourgogne. Ce sont les auteurs de ces travaux récents, dont beaucoup restent inédits, qui ont été réunis à Lyon II en mai 2003 à l'initiative de Jean-Michel Poisson (maître de conférences à l'EHESS) et Cyrille Ducourthial (doctorant de l'université de Lyon II), dans le cadre d'une table ronde, introduite et conclue par Michel Parisse et Christian Lauranson-Rosaz. Les actes de ces journées ont par la suite été rassemblés à l'université de Savoie, où ils ont été complétés par un avant-propos historiographique de Guido Castelnuovo.

4 L'unité de ce livre tient dans les perspectives monographiques qui ont été adoptées au fil des études, ce qui permet à l'ouvrage d'offrir un tableau des différents espaces du royaume de Bourgogne, à l'exception toutefois de la Provence orientale et de la Franche-Comté, dont l'historiographie n'a pas fait l'objet d'un renouvellement récent. On y trouvera ainsi les contributions d'Eliana Magnani, de Marie-Pierre Estienne et de Florian Mazel sur la Provence, celles de Pierre-Yves Laffont (Vivarais), Laurent Grimaldi (Viennois) et Laurent Ripart (Dauphiné et Savoie) sur la moyenne vallée du Rhône, tandis que le haut bassin rhodanien fait l'objet des études de Pierre Ganivet (Lyonnais) et de François Demotz (Suisse romande).

5 Ces études monographiques se polarisent sur les alentours de l'an Mil, autrement dit sur une époque qui voit apparaître de nouvelles sources offrant la possibilité d'une approche régionale. En favorisant l'écriture, mais plus encore la conservation des archives, la réforme monastique offre de nouveaux fonds à l'historien du royaume de Bourgogne, qui ne disposait guère avant le milieu du $\mathrm{X}^{\mathrm{e}}$ siècle que de très rares documents. Les chartriers et cartulaires relativement denses de Savigny, Ainay, SaintAndré-le-Bas, Saint-Maurice d'Agaune, Saint-Barnard de Romans, Lérins ou SaintVictor de Marseille apportent ainsi de nouveaux matériaux, pour l'essentiel centrés sur la vallée du Rhône et le littoral méditerranéen. Plus défavorisés, les espaces alpins, qui n'accueillaient en l'an Mil que de rares et modestes établissements monastiques, restèrent toutefois, au moins en partie, à l'écart de cette « révélation documentaire ».

6 Surtout, l'an Mil correspond en Bourgogne au règne de Rodolphe III (993-1032), autrement dit à un règne charnière qui vit l'effondrement du royaume bourguignon. Sous l'autorité d'un roi sans héritier, la monarchie rodolphienne ne put résister à la montée en puissance de l'empire germanique, dont l'autorité croissante sapa en profondeur les fondements de la royauté rodolphienne. La monarchie bourguignonne 
ne s'en releva pas et laissa la place à un royaume sans roi, organisé en une marqueterie de nouvelles principautés. Si les plus anciennes étaient déjà en place dans les dernières décennies du $\mathrm{X}^{\mathrm{e}}$ siècle (Provence, comté de Bourgogne), bien d'autres en revanche n'apparurent, souvent d'ailleurs assez brutalement, que sous le règne de Rodolphe III (Savoie, Dauphiné, etc.).

7 Dans ce contexte, il n'est guère étonnant que l'essentiel des communications de cet ouvrage soit centré sur la genèse des principautés, à l'exemple de la contribution de Florian Mazel, qui étudie le cas provençal en reconsidérant entièrement les conclusions de Jean-Pierre Poly. Pour l'essentiel, ces travaux rompent avec une vieille historiographie, centrée sur les seules dynasties princières, en mettant en exergue l'importance des institutions ecclésiastiques dans le processus de constitution des principautés. En s'émancipant de la tutelle royale, à la faveur souvent de l'autorité impériale, les établissements ecclésiastiques du royaume de Bourgogne donnèrent naissance à des noyaux de pouvoirs autonomes, dont le contrôle permit à de jeunes dynasties aristocratiques de poser les fondements de leurs pouvoirs princiers.

8 À côté de ces études d'histoires politiques, d'autres problématiques sont aussi abordées. Marie-Pierre Estienne étudie ainsi le développement des fortifications castrales dans les Baronnies, tandis qu'Eliana Magnani étudie les formes de vie consacrée dans la Provence du début du XI ${ }^{e}$ siècle: elle montre que dans un contexte marqué par la faiblesse du monachisme féminin, de nombreuses femmes issues de l'aristocratie menèrent une vie de Deo devota, dans l'entourage d'un établissement masculin.

9 Au total, cet ouvrage témoigne du renouveau, timide mais néanmoins bien réel, que connaissent actuellement les études sur le royaume de Bourgogne ${ }^{11}$. Elle met en évidence l'importance du développement dans les vingt dernières années des thèses de nouveau régime, qui ont pu apporter à la recherche historique un ensemble conséquent de nouveaux matériaux. Elle offre, enfin, au débat sur l'an Mil, un dossier jusque-là très peu pris en compte, si ce n'est pour la Provence par l'intermédiaire de Jean-Pierre Poly. De ce point de vue, les conclusions de l'ouvrage peuvent sembler paradoxales: si en Provence, l'an Mil ne semble guère, contrairement à une longue tradition historiographique, constituer une réelle césure, ce n'est pas le cas en revanche plus au nord, où la crise puis la disparition de la royauté rodolphienne modifièrent en profondeur l'organisation politique et religieuse des sociétés locales.

\section{NOTES}

1. R. POUPARDIN, Le royaume de Provence sous les Carolingiens (855-933 ?), Paris, 1901 [reprint GenèveMarseille, 1974] et ID., Le royaume de Bourgogne(888-1038), Paris, 1907 [reprint Genève, 1974].

2. É. FOURNIAL, «La souveraineté du Lyonnais au X'siècle ", Le Moyen Âge, 62 (1956), p. 413-452 et surtout par C. R. BRÜHL, Naissance de deux peuples : Français et Allemands (IX et XI siècles), Paris, 1996, p. 206-207.

3. P. FOURNIER, Le Royaume d'Arles et de Vienne (1138-1378). Étude sur la formation territoriale de la France de l'Est et du Sud-Est, Paris, 1891 ; R. PARISOT, Le royaume de Lorraine sous les Carolingiens 
(843-923), Paris, 1898 [reprint Genève, 1975] et L. JACOB, Le royaume de Bourgogne sous les empereurs franconiens (1038-1135). Essai sur la domination impériale dans l'Est et le Sud-Est de la France aux XI et XII ${ }^{\mathrm{e}}$ siècles, Paris, 1906.

4. A. HOFMEISTER, Burgund und Deutschland in früheren Mittelalter. Eine Studie über die Entstehung des Arelatischen Reiches und seine politische Bedeutung, Leipzig, 1914 [reprint Darmstadt, 1962].

5. L. DUPRAZ, «L'avènement de Rodolphe $\mathrm{I}^{\mathrm{er}}$ et la naissance du royaume de Bourgogne (6 janvier 888)", Revue d'histoire suisse, 13 (1963), p.177-195 et G. TABAcco, «Forme medievali di dominazione nelle Alpi occidentali », Bollettino storico-bibliografico subalpino, 60 (1962), p. 327-354.

6. B. BLIGNY, L'Église et les ordres religieux dans le royaume de Bourgogne aux $\mathrm{XI}^{\mathrm{e}}$ et $\mathrm{XII}{ }^{\mathrm{e}}$ siècles, Paris, 1960 (Collection des cahiers d'histoire publiée par les universités de Clermont, Lyon, Grenoble, 4) et J.-P. POLY, La Provence et la société féodale (879-1166). Contribution à l'étude des structures dites féodales dans le Midi, Paris, 1976.

7. Die Urkunden der Burgundischen Rudolfinger. Regum Burgundie e stirpe rudolfina diplomata et acta, éd. Th. SCHIEFFER, Munich, 1977 (MGH, Regum Burgundice e stirpe rudolfina diplomata et acta).

8. E. HLAWITSCHKA, «Die Verwandtschaftlichen Verbindungen zwischen dem hochburgundischen und dem niederburgundischen Königshaus. Zugleich ein Beitrag zur Geschichte Burgunds in der 1. Hälfte des 10. Jahrhunderts ", in W. SCHLÖGL et P. HERDE (dir.), Grundwissenschaften und Geschichte, Festschrift für Peter Acht, Kallmünz, 1976 (Münchener historische Studien, Abteilung geschichtliche Hilfswissenschaften, 15) [rééd.: ID., Stirps regia. Forschungen zu Königtum und Führungssichten im frühen Mittelalter. Ausgewählte Aufsätze. Festgaben zu seinem 60. Geburtstag, Francfort/Berne, 1988, p. 269-298]; B. DEVRÉGILLE, Hugues de Salins, archevêque de Besançon, 1031-1066, Lille, 1983 et R. WALPEN, Studien zur Geschichte des Wallis im Mittelalter (9 bis 15. Jahrhundert), Berne, 1983 et P. RÜcK, « Das öffentliche Kanzellariat in der Westschweiz (8-14 jh.) », Landesherrliche Kanzleien im Spätmittelalter. Referate zum VI. internationalen Kongress für Diplomatik, Munich, 1983, p. 203-271.

9. G. SERGI, « Genesi di un regno effimero : la Borgogna di Rodolfo I », Bollettino storico-bibliografico subalpino, 87 (1989), p. 5-44; ID., "Istituzioni politiche e società nel regno di Borgogna ", in Il Secolo di ferro : mito e realtà del secolo X (19-25 aprile 1990). XXXVIII settimane di studio del Centro italiano di studi sull'alto medioevo, Spolète, 1991, I, p. 205-236 ; ID., I confini del potere. Marche et signorie fra due regni medievale, Turin, 1995 ; G. CASTELNUOVo,L'aristocrazia del Vaud fino alla conquista sabauda (inizio XI-metà XIII secolo), Turin, 1990 (Biblioteca storica subalpina, 207) [trad. partielle en français: Seigneurs et lignages du pays de Vaud. Du royaume de Bourgogne à l'arrivée des Savoie, Lausanne, 1994 (Cahiers lausannois d'histoire médiévale, 11)] ; ID., "Les élites des royaumes de Bourgogne (milieu IX ${ }^{\mathrm{e}}$-milieu $\mathrm{X}^{\mathrm{e}}$ siècle) », in R. LE JAN (dir.), La royauté et les élites dans l'Europe carolingienne ( $d u$ début du IX aux environs de 920), Lille, 1998 (Centre d'histoire de l'Europe du Nord-Ouest, 17), p. 383-408 et A. BARBERO, "Conte e vescovo in Valle d'Aosta (secoli XI-XIII) ", Bollettino Storico-Bibliografico Subalpino, 86 (1988), p. 39-75 [repris in ID., Valle d'Aosta medievale, Naples, 2000 (Bibliothèque de l'Archivum Augustanum, 27), p. 1-40].

10. J.-D. MOREROD, Genèse d'une principauté épiscopale. La politique des évêques de Lausanne (IX $\mathrm{e}_{-}$ $X I V^{\mathrm{e}}$ siècle), Lausanne, 2000 (Bibliothèque historique vaudoise, 116).

11. P. PARAVY (dir.), Des Burgondes au royaume de Bourgogne ( $\mathrm{V}^{\mathrm{e}}-\mathrm{X}^{\mathrm{e}}$ siècle), Grenoble, 2002 ; F. DEMOTZ, La Bourgogne, dernier des royaumes carolingiens (855-1056) : rois, pouvoirs et élites autour du Léman, Lausanne, 2008 et M. GAILLARD, M. MARGUE et H. PETIAU (dir.), De la mer du Nord à la Méditerranée: Francia media, une région au cœur de l'Europe (c. 840-c. 1050), Actes du colloque de Metz, Luxembourg, Trêves (8-11 février 2006), sous presse. 